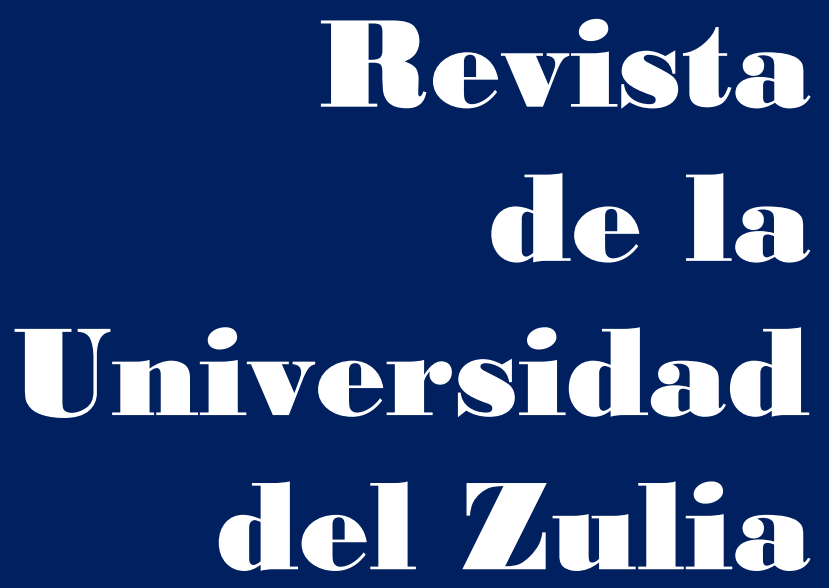

Fundada en 1947

por el Dr. Jesús Enrique Lossada

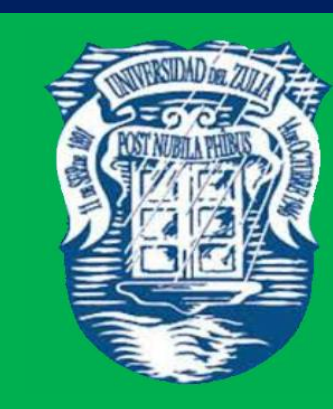

Ciencias del

Agrad,

Ingemiería

y Tecinología

\section{Aกัต 13 No $\mathbf{3 6}$} Enero - Abril 2022

Tercera Épaca

Maracailbo-Venezuela 


\title{
Satisfacción laboral en colaboradores de entidades públicas y financieras del Perú
}

\author{
Irma Victoria Martínez Nole* \\ Priscila E. Luján-Vera ** \\ Luis Ramón Trelles Pozo*** \\ Lucy Mariela García Vilela **** \\ Juan Isaías Cango Córdova $a^{* * * *}$
}

RESUMEN

La presente investigación tiene como objetivo comparar la satisfacción laboral de los colaboradores de la entidad pública Municipalidad provincial de Sullana y de la entidad financiera Caja Sullana. La metodología se centra en el enfoque cuantitativo, diseño descriptivo comparativo. La muestra estuvo constituida por 150 colaboradores, 73 del área de Desarrollo Social de la Municipalidad provincial de Sullana y 77 pertenecientes al área de talento humano de la Caja Municipal de Sullana. Para la recolección de datos se aplicó la evaluación psicométrica en ambas poblaciones, basada en el cuestionario de satisfacción laboral SL-ARG de Alex Ruiz Gómez. Entre los resultados encontrados se halló que existen diferencias significativas entre ambas entidades. Se concluye que existe un marco de diferencia entre los niveles de satisfacción de los colaboradores de la administración privada y los colaboradores que están sometidos a la administración pública.

PALABRAS CLAVE: Trabajo; gestión de personal; organización y gestión; Perú.

* Docente. Universidad Nacional de Frontera. Perú. ORCID: https://orcid.org/0000-00016570-6715. E-mail: imartinez@unf.edu.pe

**Docente Investigadora. Universidad Nacional de Frontera. Perú. ORCID: https:/orcid.org/0000-0002-1359-5715. E-mail: priscilavera930@gmail.com

*** Docente Investigador. Universidad Nacional de Frontera. Perú. ORCID: https://orcid.org/0000-0002-5523-2031. E-mail: ltrelles@unf.edu.pe

****Docente. Universidad Nacional de Frontera. Perú. ORCID: https://orcid.org/0000-00031123-0664. E-mail: lgarcía@unf.edu.pe

***** Docente. Universidad Nacional de Frontera. Perú. ORCID: https://orcid.org/00000003-4364-4634. E-mail: jcango@unf.edu.pe 


\section{Job satisfaction in collaborators of public and financial entities in Peru}

ABSTRACT

The present research aims to compare the job satisfaction of employees of the public entity Sullana Provincial Municipality and the financial entity Caja Sullana. The methodology focuses on the quantitative approach, comparative descriptive design. The sample consisted of 150 collaborators, 73 from the Social Development area of the Sullana Provincial Municipality and 77 from the Human Talent area of the Sullana Municipal Fund. For data collection, the psychometric evaluation was applied in both populations, based on the SLARG job satisfaction questionnaire by Alex Ruiz Gómez. Among the results found, it was discovered that there are significant differences between both entities. It is concluded that there is a framework of difference between the levels of satisfaction of the collaborators of the private administration and the collaborators who are submitted to the public administration.

KEY WORDS: Labour; Personnel management; Organization and management; Peru.

\section{Introducción}

La Organización Internacional del Trabajo (2020) sostiene que el mundo laboral actual se caracteriza por sus profundos déficits de trabajo decente; que se define como la suma de los deseos que las personas proyectan en su vida laboral y abarcan desde las oportunidades presentadas en el campo laboral con el otorgamiento de ingresos acordes a la preparación del empleado, las condiciones adecuadas respecto a infraestructura, como la igualdad de trato y la libertad de opinar y expresar sus ideales y emociones.

Desde que se inició el confinamiento debido a la pandemia por la COVID 19 las entidades públicas y las entidades financieras privadas asumieron el reto de continuar con las acciones laborales; pero esta vez a través de un nuevo estilo, como es el teletrabajo. Los países que aún no habían experimentado este sistema tuvieron que implementarlo por la misma necesidad de las empresas en continuar ofreciendo productos y servicios a sus clientes y mantener así, el dinamismo de su economía. Es entonces que, en el Perú, el sector privado se adaptó a este estilo de trabajo de manera inmediata; en paralelo el sector público siguió los mismos pasos, pero con significativas deficiencias. 
Cabe precisar que el teletrabajo se conceptualiza como la forma de ejercer las funciones laborales fuera de las instalaciones de la empresa o el empleador, haciendo utilidad de las herramientas tecnológicas como: tabletas, móviles, computadores, laptops, teléfono, plataformas virtuales e incluso redes sociales. Y este estilo de trabajo se da en el marco de acuerdos en común entre el empleado y el empleador mediante un contrato, con fines específicos de continuar con los objetivos trazados por la organización.

En el Perú se ha percibido información estadística correspondiente a la expectativa en el trabajo: aproximadamente el 50\% de los trabajadores no se encuentra contento dentro de su ambiente laboral, afectando con esto su capacidad de responsabilidad y compromiso con las funciones y trabajos estipulados. Asimismo, el 45\% de la población de trabajadores que participaron en una investigación realizada, manifestó que no se siente feliz con su trabajo actual, siendo el principal motivo de dicha situación, los problemas relacionados con un inadecuado clima laboral (Lira, 2014).

En nuestra localidad (Sullana) se puede apreciar que existen instituciones públicas que no cuentan con instalaciones adecuadas y acordes a la necesidad de los trabajadores, ya que carecen de las condiciones para que se pueda realizar el cumplimiento de una jornada laboral completa. Más aún, con el sistema virtual, la documentación del usuario queda transitando en la nube a la espera de una atención inmediata, caracterizando de deficiente el desempeño del sistema; a diferencia de lo propuesto por las entidades privadas que cuentan con todo tipo de recursos para el desarrollo laboral, y que han implementado sistemas virtuales de atención inmediata con la visión de mantenerse en el mercado y salvaguardar su clientela.

En base a los expuesto, la investigación tuvo como objetivo primordial comparar la satisfacción laboral de los colaboradores de la entidad pública Municipalidad provincial de Sullana y de la entidad financiera Caja Sullana.

\section{Satisfacción laboral}

Los investigadores Yáñez et al. (2010) sostienen que la satisfacción laboral es el estado emocional positivo que emerge del resultado de la experiencia en el campo laboral de un empleado. Es así que, Robbins (2004) define la satisfacción laboral como la actitud global del individuo respecto a su espacio laboral, lo que implica que un colaborador con elevado 
REVISTA DE LA UNIVERSIDAD DEL ZULIA. $3^{a}$ época. Año $13 \mathrm{~N}^{\circ}$ 36, 2022

Irma Victoria Martínez Nole et al. /// Satisfacción laboral en colaboradores de entidades públicas ... 388-399

DOI: http://dx.doi.org/10.46925//rdluz.36.25

nivel de satisfacción tiende a tener actitudes positivas que lo llevarán a tener mayor productividad, mientras aquella que se sienta insatisfecha acogerá actitudes negativas.

Para Coronel et al. (2020) la satisfacción de los integrantes de un grupo demanda trabajo colaborativo y comunicación, con metas en común por cumplir. Así mismo, Bernal y Flores (2020) afirman que la satisfacción del personal en base a su espacio laboral se vuelve un aspecto central para las organizaciones, ya que se ha reflejado que la satisfacción en el trabajo es una condicionante en el desenvolvimiento del colaborador que posteriormente se evidenciara en la satisfacción del usuario y la calidad de los servicios ofertados.

Pizarro et al. (2019) afirman que la motivación es un elemento indispensable que todas las personas deben poseer para la lograr objetivos en diferentes campos de la vida. Para García et al. (2020) la motivación laboral en las instituciones académicas es un factor determinante en la satisfacción laboral, convirtiéndose en esencial a la hora de ejecutar actividades encargadas dentro de la organización, para mantener un ambiente cálido e interactivo. Es así, que el colaborador necesita herramientas e instrumentos que lo motiven y le generen placer en el desarrollo de su trabajo para que pueda alcanzar el éxito en la organización donde se desempeña.

Es preciso resaltar que Muñoz (1990), expone que la satisfacción laboral y personal es de suma relevancia en la constitución del clima organizacional como parte integral del comportamiento de la misma, reflejados en la mejora continua de los procesos productivos. Los investigadores Alva y Juárez (2014) refieren que la actividad de un empleado va más allá de ordenar files o estar a la expectativa de los usuarios, considerando la necesidad de interacción que debe existir con los jefes inmediatos, quienes serán los evaluadores del ejercicio laboral realizado en un área específica.

Moreno et al. (2018) plantean ciertos factores para alcanzar la satisfacción laboral como: la libertad en el desempeño del trabajo, la variedad de diversificación, el pago, el reconocimiento al desempeño, la flexibilidad, las capacitaciones y especializaciones; así mismo, la comunicación con los directivos como factor primordial de las buenas relaciones, todos estos componentes forman y sostienen la satisfacción en el colaborador.

Por otro lado, la teoría de factores planteada por Ruiz y Zavaleta (2017) considera que la satisfacción laboral es la actitud demostrada por el trabajador ante su puesto de trabajo. 
REVISTA DE LA UNIVERSIDAD DEL ZULIA. $3^{a}$ época. Año $13 \mathrm{~N}^{\circ}$ 36, 2022

Irma Victoria Martínez Nole et al. /// Satisfacción laboral en colaboradores de entidades públicas ... 388-399

DOI: http://dx.doi.org/10.46925//rdluz.36.25

En consecuencia, para que pueda ser medible se deben evaluar diversos factores, los mismos que se detallan a continuación.

\subsection{Condiciones de trabajo}

Las condiciones de trabajo están enmarcadas como el conjunto de acciones y características materiales que engloban el sistema político, social, cultural, económico, ambiental y organizacional, donde sucede la interacción laboral. Investigaciones han sostenido que la estructura de estas condiciones incide en la calidad laboral de cada colaborador e incluso en su misma vida personal, donde hasta cierto punto puede comprometer la salud física y mental (Martínez, Oviedo y Luna, 2016).

Para Quezada et al. (2021) las condiciones laborales se relacionan con los recursos financieros que posee la entidad, otorgando la seguridad y el respaldo al empleado de ser remunerado en el tiempo que estipula su contrato, así mismo, la adquisición de equipos que permitirá el ahorro de tiempo y la entrega de actividades en el lapso indicado. En tal sentido, Cacua, Carvajal y Hernández (2017) sostienen que las condiciones laborales son relevantes para el avance económico de una organización e institución, y tan solo el hecho de pensar en su abandono genera poblaciones vulnerables a la transformación económica donde los estilos de calidad de vida podrían verse comprometidos.

\subsection{Remuneración y beneficios}

\subsubsection{Remuneración}

La remuneración es el pago efectivo que se realiza a un colaborador por ejercer acciones enmarcadas en un conjunto de normas establecidas por una institución o entidad, que conlleva al funcionamiento de los procesos y al cumplimiento de objetivos de la organización. El Decreto Legislativo N 728, Ley de Productividad y Competitividad Laboral en el Perú, constituye que la "remuneración es todo lo que el trabajador recibe por sus servicios, en dinero o en especie, cualquiera sea la forma o denominación que se le dé, siempre que sea de su libre disposición". En tal sentido, es lo que por derecho le corresponde a un empleado por el servicio ofrecido.

\subsubsection{Beneficios}


REVISTA DE LA UNIVERSIDAD DEL ZULIA. $3^{a}$ época. Año $13 \mathrm{~N}^{\circ}$ 36, 2022

Irma Victoria Martínez Nole et al. /// Satisfacción laboral en colaboradores de entidades públicas ... 388-399

DOI: http://dx.doi.org/10.46925//rdluz.36.25

Manzo y Moncallo (2004) sostienen que "los beneficios son aquellas facilidades, comodidades, ventajas y servicios que las empresas ofrecen para ahorrarles esfuerzos y preocupaciones, que pueden ser financiadas parcial o totalmente. Estos beneficios constituyen medios indispensables para el mantenimiento de la fuerza laboral dentro de un nivel satisfactorio de moral y productividad".

\subsection{Relaciones humanas}

Caicedo et al. (2018) sostienen que el ser humano, desde su existencia, siempre ha tenido la necesidad de relacionarse con su entorno, generar grupos amicales como laborales que le han permitido dinamizar su rendimiento y habilidad en las actividades encomendadas, aprovechando eficientemente los recursos otorgados en el campo laboral. Así mismo, Ismail, Karkoulian y Kertechian (2019) argumentan que los estrategas, al considerar las relaciones humanas, deben entender cómo conseguir que los colaboradores estén contentos e incentivados en el espacio laboral, considerando que, a mayor satisfacción, se obtendrá resultados eficaces en las tareas asignadas.

\section{Metodología}

La investigación responde a las características del diseño no experimental de tipo transaccional, denominado diseño descriptivo comparativo; este diseño según Hernández, Fernández, \& Baptista (2014) se describe como la consolidación de dos o más investigaciones de tipo descriptivas simples, ya que consiste en recolectar los datos relevantes referentes a un mismo fenómeno de más de una muestra poblacional, para posteriormente realizar la comparación respectiva de los datos hallados.

El diseño para la ejecución del presente estudio es el siguiente:

-Ml: Colaboradores del área de recursos humanos de la Caja Sullana.

-M2: Colaboradores del área de desarrollo social de la Municipalidad Provincial de Sullana. -Ol y O2: Medición de los niveles de satisfacción laboral.

La muestra de estudio estuvo constituida por 150 colaboradores de dos subgerencias de la Caja Sullana y por 6 subgerencias del área de Desarrollo Social de la Municipalidad Provincial de Sullana, como se muestra a continuación: 
REVISTA DE LA UNIVERSIDAD DEL ZULIA. $3^{a}$ época. Año $13 \mathrm{~N}^{\circ}$ 36, 2022

Irma Victoria Martínez Nole et al. /// Satisfacción laboral en colaboradores de entidades públicas ... 388-399

DOI: http://dx.doi.org/10.46925//rdluz.36.25

Tabla 1. Muestra de estudio.

\begin{tabular}{cccc}
\hline $\begin{array}{c}\text { Sub gerencias área de Gestión } \\
\text { del Talento Humano }\end{array}$ & Cantidad & $\begin{array}{c}\text { Subgerencias área de } \\
\text { Desarrollo Social }\end{array}$ & Cantidad \\
\hline $\begin{array}{c}\text { Gestión de desarrollo } \\
\text { Humano }\end{array}$ & 44 & Educación y Cultura & 15 \\
Capacitación & 33 & Responsabilidad Social & 09 \\
& & Registro Civil & 16 \\
& & Programas Alimentarios & 17 \\
TOTAL & 77 & OMAPED & 13 \\
& & TOTAL & 73 \\
\hline
\end{tabular}

Fuente: Elaboración Propia.

3. Resultados y Discusión

Tabla 2. Diferencias de satisfacción laboral entre ambas entidades.

\begin{tabular}{|c|c|c|c|c|c|c|c|c|c|}
\hline \multicolumn{10}{|c|}{ Muestras independientes } \\
\hline \multirow[b]{2}{*}{ Satisfacción Laboral } & \multicolumn{2}{|c|}{$\begin{array}{c}\text { Prueba de } \\
\text { Levene de } \\
\text { igualdad de } \\
\text { varianzas }\end{array}$} & \multicolumn{7}{|c|}{ Prueba t para la igualdad de medias } \\
\hline & F & Sig. & $\mathrm{t}$ & $\mathrm{gl}$ & $\underset{\text { (bilateral) }}{\text { Sig. }}$ & $\begin{array}{c}\text { Diferencia } \\
\text { de } \\
\text { medias }\end{array}$ & $\begin{array}{l}\text { Diferencia } \\
\text { de error } \\
\text { estándar }\end{array}$ & $\begin{array}{r}95 \% \\
\text { interv } \\
\text { confian } \\
\text { difer } \\
\text { Inferior }\end{array}$ & $\begin{array}{l}\% \text { de } \\
\text { valo de } \\
\text { nza de la } \\
\text { rencia } \\
\text { Superior }\end{array}$ \\
\hline $\begin{array}{l}\text { Se asumen varianzas } \\
\text { iguales }\end{array}$ & 410,916 &, 000 & 14,057 & 148 &, 000 & 58,571 & 4,167 & 50,337 & 66,805 \\
\hline $\begin{array}{l}\text { No se asumen varianzas } \\
\text { iguales }\end{array}$ & & & 13,698 & 74,622 &, 000 & 58,571 & 4,276 & 50,052 & 67,089 \\
\hline
\end{tabular}

Fuente: Elaboración Propia

En la tabla 2 se evidencia que ante la prueba $T$ y de Levene para la igualdad de medias y varianzas respectivamente; se aprecia que las medias de ambos grupos son distintas ya que la diferencia de las mismas asume un valor de 58,57. Así mismo, se aprecia que, al ser el valor de significancia, en ambas pruebas, menor a 0.05 indica que si existe diferencia entre las varianzas. 
REVISTA DE LA UNIVERSIDAD DEL ZULIA. 3a época. Año $13 \mathrm{~N}^{\circ}$ 36, 2022

Irma Victoria Martínez Nole et al. /// Satisfacción laboral en colaboradores de entidades públicas ... 388-399

DOI: http://dx.doi.org/10.46925//rdluz.36.25

Tabla 3. Condiciones de trabajo.

\begin{tabular}{|c|c|c|c|c|c|c|c|c|c|}
\hline \multirow{3}{*}{$\begin{array}{c}\text { Factor I: } \\
\text { Condiciones de Trabajo }\end{array}$} & \multicolumn{2}{|c|}{$\begin{array}{l}\text { Prueba de Levene } \\
\text { de igualdad de } \\
\text { varianzas }\end{array}$} & \multicolumn{7}{|c|}{ Prueba t para la igualdad de medias } \\
\hline & \multirow[t]{2}{*}{$\mathrm{F}$} & \multirow[t]{2}{*}{ Sig. } & \multirow[t]{2}{*}{$\mathrm{t}$} & \multirow[t]{2}{*}{$\mathrm{gl}$} & \multirow[t]{2}{*}{$\begin{array}{c}\text { Sig. } \\
\text { (bilateral) }\end{array}$} & \multirow[t]{2}{*}{$\begin{array}{l}\text { Diferencia } \\
\text { de medias }\end{array}$} & \multirow[t]{2}{*}{$\begin{array}{l}\text { Diferencia } \\
\text { de error } \\
\text { estándar }\end{array}$} & \multicolumn{2}{|c|}{$\begin{array}{l}95 \% \text { de intervalo de } \\
\text { confianza de la } \\
\text { diferencia }\end{array}$} \\
\hline & & & & & & & & Inferior & Superior \\
\hline Se asumen varianzas iguales & 272,917 & ,000 & 14,417 & 148 & ,000 & 15,244 & 1,057 & 13,154 & 17,333 \\
\hline No se asumen varianzas iguales & & & 14,049 & 74,511 & ,000 & 15,244 & 1,085 & 13,082 & 17,405 \\
\hline
\end{tabular}

En la tabla 3 se evidencia que ante la prueba $T$ y de Levene para la igualdad de medias y varianzas respectivamente; las medias de ambos grupos son distintas ya que la diferencia de las mismas asume un valor de 15,244. Se apreciar que, al ser el valor de significancia, en ambas pruebas, menor a 0.05 indica que si existe diferencia entre las varianzas. Estadísticamente también se evidencia una desigualdad significativa entre la media y la varianza de esta dimensión, dándonos como resultado que existen diferencias importantes entre los grupos. Esto permite argumentar que las condiciones de trabajo percibidas por los colaboradores de una entidad privada son diferentes a las de los colaboradores de una entidad pública, en la medida que los trabajadores de la entidad privada se sienten más retribuidos con los materiales necesarios para el buen desempeño laboral. Esto es ratificado por Robbins (2004) quien indica que uno de los aspectos importantes que alimentan la satisfacción laboral es la recompensa equitativa y las condiciones de trabajo que se convierten en un respaldo para que este pueda responder de manera apropiada a su desempeño laboral, llevándolo esto a un buen nivel de productividad.

Tabla 4. Remuneración y beneficios.

\section{Prueba de muestras independientes}

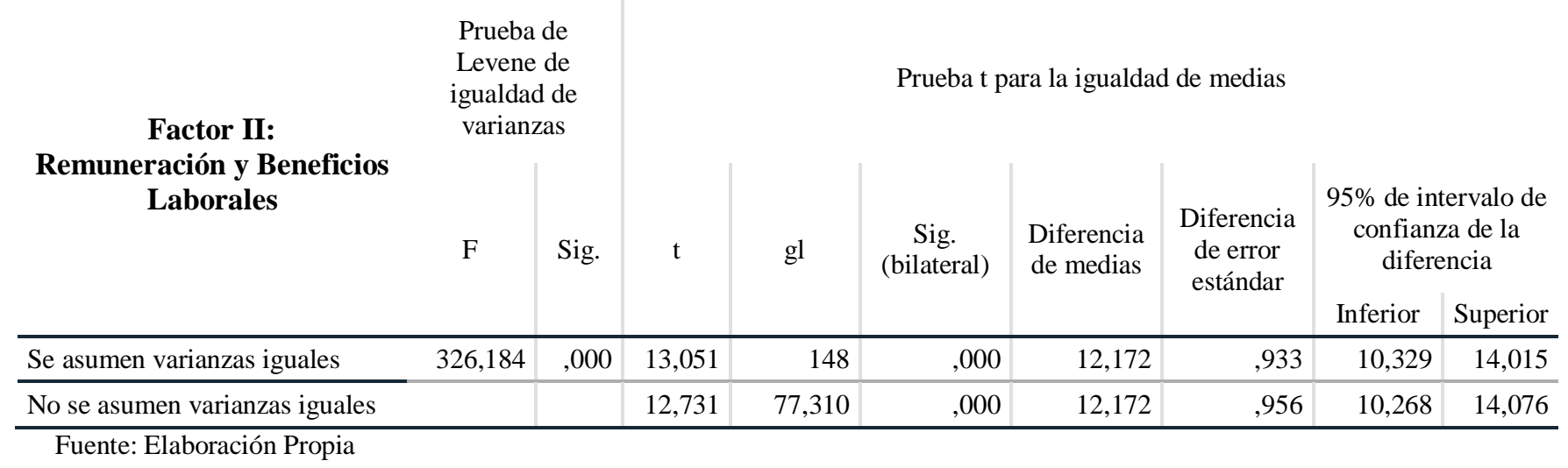


REVISTA DE LA UNIVERSIDAD DEL ZULIA. $3^{a}$ época. Año $13 \mathrm{~N}^{\circ}$ 36, 2022 Irma Victoria Martínez Nole et al. /// Satisfacción laboral en colaboradores de entidades públicas ... 388-399 DOI: http://dx.doi.org/10.46925//rdluz.36.25

En la tabla 4 se evidencia que ante la prueba $T$ y de Levene para la igualdad de medias y varianzas respectivamente; las medias de ambos grupos son distintas ya que la diferencia de las mismas asume un valor de 12,172. Así mismo, se observa que, al ser el valor de significancia, en ambas pruebas, menor a 0.05 indica que si existe diferencia entre las varianzas. Esto permite argumentar que beneficios y bonificaciones de trabajo percibidas por los colaboradores de una entidad privada son diferentes a las de los colaboradores de una entidad pública. Esto es validado con los hallazgos realizados por Alva y Juárez (2014), quienes al desarrollar una investigación en una empresa privada de Trujillo llegaron a la conclusión que uno de los factores importantes en toda entidad es la retribución económica, ya que, pese a que varios de sus colaboradores eran compensados con días libres al ser convocados a trabajar un día feriado, no se mostraban conformes debido a que esperaban una retribución económica acorde a su esfuerzo y compromiso.

Tabla 5. Relaciones humanas.

Prueba de muestras independientes

\begin{tabular}{|c|c|c|c|c|c|c|c|c|c|}
\hline \multirow{3}{*}{$\begin{array}{c}\text { Factor IV: } \\
\text { Recursos Humanos }\end{array}$} & \multicolumn{2}{|c|}{$\begin{array}{l}\text { Prueba de } \\
\text { Levene de } \\
\text { igualdad de } \\
\text { varianzas }\end{array}$} & \multicolumn{7}{|c|}{ Prueba t para la igualdad de medias } \\
\hline & \multirow[t]{2}{*}{$\mathrm{F}$} & \multirow[t]{2}{*}{ Sig. } & \multirow[t]{2}{*}{$\mathrm{t}$} & \multirow[t]{2}{*}{$\mathrm{gl}$} & \multirow[t]{2}{*}{$\begin{array}{l}\text { Sig. } \\
\text { (bilateral) }\end{array}$} & \multirow[t]{2}{*}{$\begin{array}{l}\text { Diferencia } \\
\text { de medias }\end{array}$} & \multirow[t]{2}{*}{$\begin{array}{l}\text { Diferencia } \\
\text { de error } \\
\text { estándar }\end{array}$} & \multicolumn{2}{|c|}{$\begin{array}{l}95 \% \text { de intervalo de } \\
\text { confianza de la } \\
\text { diferencia }\end{array}$} \\
\hline & & & & & & & & Inferior & Superior \\
\hline Se asumen varianzas iguales & 11,331 & ,001 & 9,258 & 148 & 000 & 3,446 & ,372 & 2,710 & 4,182 \\
\hline No se asumen varianzas iguales & & & 9,151 & 120,122 & 000 & 3,446 & 377 & 2,700 & 4,192 \\
\hline
\end{tabular}

Fuente: Elaboración Propia

En la tabla 5 se muestra que ante la prueba T y de Levene para la igualdad de medias y varianzas respectivamente, las medias de ambos grupos son diferentes ya que la diferencia de las mismas asume un valor de 3,446. Se aprecia que, al ser el valor de significancia, en ambas pruebas, menor a 0.05 indica que si existe diferencia entre las varianzas. En esta dimensión se refleja que existe un 49\% de parcial satisfacción por parte de los colaboradores de la Caja Sullana en contraparte a los colaboradores de la Municipalidad Provincial de Sullana, en donde se encuentra un 36 y 34\% de regular y parcial insatisfacción. De igual forma, se evidencia una desigualdad significativa entre la media y la varianza de esta dimensión, dándonos como resultado que existen diferencias importantes entre ambos grupos. Ante lo 
REVISTA DE LA UNIVERSIDAD DEL ZULIA. $3^{a}$ época. Año $13 \mathrm{~N}^{\circ}$ 36, 2022

Irma Victoria Martínez Nole et al. /// Satisfacción laboral en colaboradores de entidades públicas ... 388-399

DOI: http://dx.doi.org/10.46925//rdluz.36.25

hallado podemos determinar que para los colaboradores de la Caja Sullana existe cierto nivel de agrado en el establecimiento de las relaciones fraternales, confianza y empatía dentro de su línea laboral; mientras que para los colaboradores de la Municipalidad provincial existe cierto rechazo en base a los bajos niveles de satisfacción presentados. Esto es complementado con lo planteado por Mc Clelland (1973), en su teoría denominada teoría de las necesidades, quien sostiene que existen tres necesidades básicas que tiene el ser humano y se desarrollan en base al aprendizaje y el contacto con la experiencia que determinan la satisfacción e insatisfacción laboral, entre ellas tenemos: la necesidad de afiliación que se basa en la capacidad del ser humano de brindar afecto, amor y una buena interacción con la sociedad, prefiriendo satisfacer las necesidades sociales, familiares y afectivas.

Tabla 6. Desarrollo percibido por los colaboradores de ambas entidades.

\begin{tabular}{|c|c|c|c|c|c|c|c|c|}
\hline & rueba & e mues & $s$ indep & ndientes & & & & \\
\hline $\begin{array}{r}\text { Prueba de } 1 \\
\text { de iguald } \\
\text { varian }\end{array}$ & $\begin{array}{l}\text { evene } \\
\text { d de } \\
\text { as }\end{array}$ & & & Prueba & para la igual & dd de medias & & \\
\hline $\mathrm{F}$ & Sig. & $\mathrm{t}$ & $\mathrm{gl}$ & $\begin{array}{c}\text { Sig. } \\
\text { (bilateral) }\end{array}$ & $\begin{array}{l}\text { Diferencia } \\
\text { de medias }\end{array}$ & $\begin{array}{l}\text { Diferencia } \\
\text { de error }\end{array}$ & $\begin{array}{r}95 \% \text { de in } \\
\text { confian } \\
\text { difer }\end{array}$ & $\begin{array}{l}\text { ervalo de } \\
\text { a de la } \\
\text { ncia }\end{array}$ \\
\hline & & & & & & & Inferior & Superior \\
\hline 177,454 & ,000 & 12,749 & 148 & ,000 & 8,976 & ,704 & 7,585 & 10,367 \\
\hline & & 12,443 & 78,985 & ,000 & 8,976 & ,721 & 7,540 & 10,412 \\
\hline
\end{tabular}

Fuente: Elaboración Propia

En la tabla 6 se observa que ante la prueba $T$ y de Levene para la igualdad de medias y varianzas respectivamente; las medias de ambos grupos son distintos ya que la diferencia de las mismas asume un valor de 8,976. Se puede evidenciar que, al ser el valor de significancia, en ambas pruebas, menor a 0.05 indica que si existe diferencia entre las varianzas. En esta dimensión se refleja una desigualdad significativa entre la media y la varianza de esta dimensión, dándonos como resultado que existen diferencias importantes entre ambos grupos. Esto nos lleva a inferir que la mayoría de los colaboradores de la Caja Sullana se muestran con expectativas de crecimiento y desarrollo profesional dentro de su campo laboral a diferencia de los colaboradores de la Municipalidad provincial de Sullana que no perciben muestras de ascenso ni de perfeccionamiento profesional por parte de su institución. Este hallazgo está relacionado con lo mencionado por Mc Clelland (1973) en su 
REVISTA DE LA UNIVERSIDAD DEL ZULIA. $3^{a}$ época. Año $13 \mathrm{~N}^{\circ}$ 36, 2022

Irma Victoria Martínez Nole et al. /// Satisfacción laboral en colaboradores de entidades públicas ... 388-399

DOI: http://dx.doi.org/10.46925//rdluz.36.25

teoría anteriormente descrita, en donde una de las necesidades presentadas por el ser humano para lograr la satisfacción laboral es la necesidad de logro que se refleja en el afán por alcanzar los objetivos y demostrar su competencia que lo llevará a su reconocimiento.

\section{Conclusiones}

Existe un marco de diferencia entre los niveles de satisfacción de los colaboradores de las instituciones de administración privada y los colaboradores que están sometidos a la administración pública, en la medida que esto genera buena disposición de compromiso ante el ejercicio laboral, y con ello, buena disposición para la atención al público y ofrecimiento del servicio.

Es indispensable que las entidades públicas y privadas generen climas organizacionales adecuados y óptimos que permitan el desarrollo de los colaboradores; así mismo implementar oportunidades de crecimiento profesional que promuevan remuneraciones adecuadas a las especializaciones de cada colaborador, en el marco de la normatividad de la instituciones donde aplican sus funciones laborales; esto con el fin de generar satisfacción laboral en ellos, lo que conducirá a obtener mayor productividad en las actividades que ejercen, y cuya réplica se verá reflejada en clientes satisfechos con los servicios que las entidades brindan.

\section{Referencias}

Alva, J. y Juárez, J. (2014). Relación entre el nivel de satisfacción laboral y el nivel de productividad de los colaboradores de la empresa Chimu Agropecuaria S.A. del distrito de Trujillo - 2014. Tesis de pregrado. Universidad Privada Antenor Orrego. Perú.

Bernal, I., \& Flores, A. J. (2020). Satisfacción laboral del capital humano en organizaciones del sector salud. Revista Venezolana De Gerencia, 25(4), 276-296.

Cacua, L., Carvajal, H. y Hernández, N. (2017). Condiciones de trabajo y su repercusión en la salud de los trabajadores de la plaza de mercado la Nueva Sexta, Cúcuta. Revista Psicoespacios, Vol. 11, N. 19, pp. 99-119. DOI: https://doi.org/10.25057/issn.2145-2776

Caicedo, L., Corrales, R., y Quintana, A. (2018). La comunicación y las relaciones humanas en el proceso de enseñanza aprendizaje. Varona. Revista Científico Metodológica, 66 (1), 1-5.

Coronel, J. W., Ayay, G., \& Milla, M. E. (2020). Clima organizacional, satisfacción laboral y gestión administrativa en los órganos de apoyo de una universidad pública. Revista De La Universidad Del Zulia, 11(31), 52-70. DOI: https://doi.org/10.46925//rdluz.31.05 
REVISTA DE LA UNIVERSIDAD DEL ZULIA. $3^{a}$ época. Año $13 \mathrm{~N}^{\circ}$ 36, 2022

Irma Victoria Martínez Nole et al. /// Satisfacción laboral en colaboradores de entidades públicas ... 388-399

DOI: http://dx.doi.org/10.46925//rdluz.36.25

Decreto Legislativo N 728, Ley de Productividad y Competitividad Laboral.

García, L. F., Fulquez, S. C., \&e Vázquez, J. (2020). Satisfacción laboral en docentes del sistema público en México: factores estresores que afectan la salud laboral. Revista Venezolana De Gerencia, 25(4), 235-246.

Hernández, R., Fernández, C., \& Baptista, M. (2014). Metodología de la investigación (6a ed.). México: Mc Graw Hill.

Ismail, H. N., Karkoulian, S., \& Kertechian, S. K. (2019). Which personal values matter most? Job performance and job satisfaction across job categories. International Journal of Organizational Analysis, 27(1), 109-124.

Lira, J. (18 de agosto del 2014). El 45\% de los trabajadores no es feliz en su centro de trabajo. Diario Gestión.

Manzo y Moncallo (2004). Remuneración salarial recibida, beneficios recibidos y aspirados relacionados con la calidad de vida laboral percibida. Tesis de pregrado. Universidad Católica Andrés Bello. Carcas-Venezuela.

Martínez, L., Oviedo, O. y Luna, C. (2013). Condiciones de Trabajo que impactan la vida laboral. Salud Uninorte, 29(3), 542-560.

MacClelland, David C. (1953). The Achivement Motive. Nueva York: Appleton-Century-Crofts

Moreno, A. V., Chang, E., \& Romero de Cuba, J. (2018). Satisfacción Laboral en las Pyme colombianas del sector Textil-Confecciónl. Revista Venezolana De Gerencia,23(82), 392-406. DOI: https://doi.org/10.37960/revista.v23i82.23755

Muñoz, A. (1990). Satisfacción e insatisfacción en el trabajo. Tesis doctoral. Facultad de Psicología, Universidad Complutense de Madrid.

Pizarro, J., Fredes, D., Inostroza, C., \&e Torreblanca, E. (2019). Motivación, satisfacción laboral y estado de flow en los trabajadores de la salud. Revista Venezolana de Gerencia, 24(87), 843-859. DOI: https://doi.org/10.37960/revista.v24i87.24640

Organización Internacional del Trabajo (2020). Perspectivas sociales y del empleo el mundo.

Quezada, M. del P., Castro, M. del P., Dios, C. A., \& Quezada, G. A. (2021). Condiciones laborales en la educación universitaria peruana: Virtualización ante la pandemia COVID -19. Revista Venezolana De Gerencia, 26(93), 110-123. DOI: https://doi.org/10.52080/rvg93.09

Robbins, S. (2004). Comportamiento organizacional. Editorial: Pearson Educación. México

Ruíz, A., Zavaleta, M. y Ruíz, P. (2017). Test de satisfacción laboral SL-ARG. Chimbote: Fondo Editorial de la Universidad César Vallejo.

Yañez, R., Arenas, M. y Ripoll Novales (2010). El impacto de las relaciones interpersonales en la satisfacción laboral general. Liberabit, 16 (2), 1993-2002. 